\title{
Temperature Dependence of the Reaction Between the Hydroxyl Radical and Organic Matter
}

Garrett McKay ${ }^{1}$, Mei Mei Dong ${ }^{2}$, Jonathan L. Kleinman ${ }^{1}$, Stephen P. Mezyk ${ }^{1}$ and Fernando L. Rosario-Ortiz $^{2, *}$

${ }^{1}$ Department of Chemistry and Biochemistry, California State University at Long Beach, 1250 Bellflower Blvd., Long Beach, CA, 90840, USA

${ }^{2}$ Department of Civil, Environmental and Architectural Engineering, 428 UCB, University of Colorado at Boulder, Boulder, CO 80309, USA

* Corresponding author: Fernando.Rosario@colorado.edu; Phone: 303-492-7607 (1)

(3)

molecular weight from SEC, SEC for EfOM samples, NOM isolate characterization data, estimated average molecular weights, a discussion of the temperature dependence of competing reactions, and all temperature dependent $k_{\mathrm{OM}-\mathrm{HO}}$ measured in this study.

Estimation of Average Molecular Weight (AMW). AMW was estimated by integrating the SEC from 100 to $18,000 \mathrm{Da}$. The lower limit of $100 \mathrm{Da}$ was chosen because no polyethylene glycols below 100 Da were used in SEC calibration, and an interference from nitrite in the EfOM samples. Though this is an approximate estimation of AMW (Equation 1), previous work by our group and others has utilized the same calibration method; thus, allowing for comparison.

In this Supplemental Information, we have included the following: estimation of average 
Temperature Dependent Rate Constants for the Reaction of $\mathrm{HO}$ - with $\mathrm{HCO}_{3}{ }^{-}, \mathrm{CO}_{3}{ }^{2-}, \mathrm{NH}_{3}$, and $\mathbf{S C N}^{-}$. Temperature dependent rate constants were used for all hydroxyl radical scavengers at each temperatures are shown below [1-3]. The Arrhenius form of the competing reactions are:

$$
\mathrm{HO}+\mathrm{SCN}^{-}\left(+\mathrm{SCN}^{-}\right) \rightarrow(\mathrm{SCN})_{2}{ }^{--} \quad k_{\mathrm{SCN}^{-}}=7.24 \times 10^{12} \exp \left(-15,800 / \mathrm{RT}^{-} \mathrm{M}^{-1} \mathrm{~s}^{-1}\right.
$$

$$
\begin{array}{ll}
\mathrm{HO}+\mathrm{HCO}_{3}{ }^{-} \rightarrow \mathrm{H}_{2} \mathrm{O}+\mathrm{CO}_{3}{ }^{-} & k_{\mathrm{HCO}_{3}}{ }^{-}=6.21 \times 10^{10} \exp \left(-21,200 / \mathrm{RT}_{)} \mathrm{M}^{-1} \mathrm{~s}^{-1}\right. \\
\mathrm{HO}+\mathrm{CO}_{3}{ }^{2-} \rightarrow \mathrm{HO}^{-}+\mathrm{CO}_{3}{ }^{-\bullet} & k_{\mathrm{CO}^{2-}}{ }^{2-}=6.68 \times 10^{12} \exp \left(-23,600 / \mathrm{RT}_{)} \mathrm{M}^{-1} \mathrm{~s}^{-1}\right.
\end{array}
$$

0

2

$$
\mathrm{HO} \cdot+\mathrm{NH}_{3} \rightarrow \mathrm{H}_{2} \mathrm{O}+{ }^{\circ} \mathrm{NH}_{2} \quad k_{\mathrm{NH} 3}=9.91 \times 10^{8} \exp (-5700 / \mathrm{RT}) \mathrm{M}^{-1} \mathrm{~s}^{-1}
$$

Using the measured temperature during experiments, and with $\mathrm{R}$ as $8.314 \mathrm{~J} \mathrm{~mol}^{-1} \mathrm{~K}^{-1}$, we were able to calculate the temperature dependent scavenging rates of all non-OM species. These temperature dependent scavenging rates were then subtracted from the observed pseudo first order rate constant $\mathrm{k}[\mathrm{X}]\left(\right.$ where $\left.[\mathrm{X}]=\left[\mathrm{HCO}_{3}{ }^{-}\right]+\left[\mathrm{CO}_{3}{ }^{2-}\right]+\left[\mathrm{NH}_{3}\right]+[\mathrm{OM}]\right)$ to yield the pseudo first order rate constant $\mathrm{k}[\mathrm{OM}]$, from which the molar carbon concentration of OM was factored out to give the bimolecular reaction rate constant, $k_{\mathrm{OM}-\mathrm{HO} .}$

0


Table S. 1. Characterization Data of DOM Isolates Used ${ }^{a}$.

\begin{tabular}{|c|c|c|c|}
\hline Sample & ESHA & SRFA & PLFA \\
\hline Cat. No. & $1 \mathrm{~S} 102 \mathrm{H}$ & $1 \mathrm{~S} 101 \mathrm{~F}$ & 1R109F \\
\hline$\% \mathrm{H}_{2} \mathrm{O}$ & 8.2 & 8.8 & 0.32 \\
\hline$\%$ Ash & 0.88 & 0.46 & 1.25 \\
\hline$\% \mathrm{C}$ & 58.13 & 52.44 & 52.47 \\
\hline$\% \mathrm{H}$ & 3.68 & 4.31 & 5.39 \\
\hline$\% \mathrm{O}$ & 34.08 & 42.2 & 31.38 \\
\hline$\% \mathrm{~N}$ & 4.14 & 0.72 & 6.51 \\
\hline \% Carboxyl & 18 & 20 & 17 \\
\hline$\%$ Aromatic & 50 & 24 & 12 \\
\hline$\%$ Heteroaliphatic & 6 & 11 & 8 \\
\hline$\%$ Aliphatic & 16 & 33 & 61 \\
\hline
\end{tabular}

$44{ }^{a}$ Data obtained from International Humic Substances Society (IHSS) website

45 (http://www.ihss.gatech.edu/).

46

47 


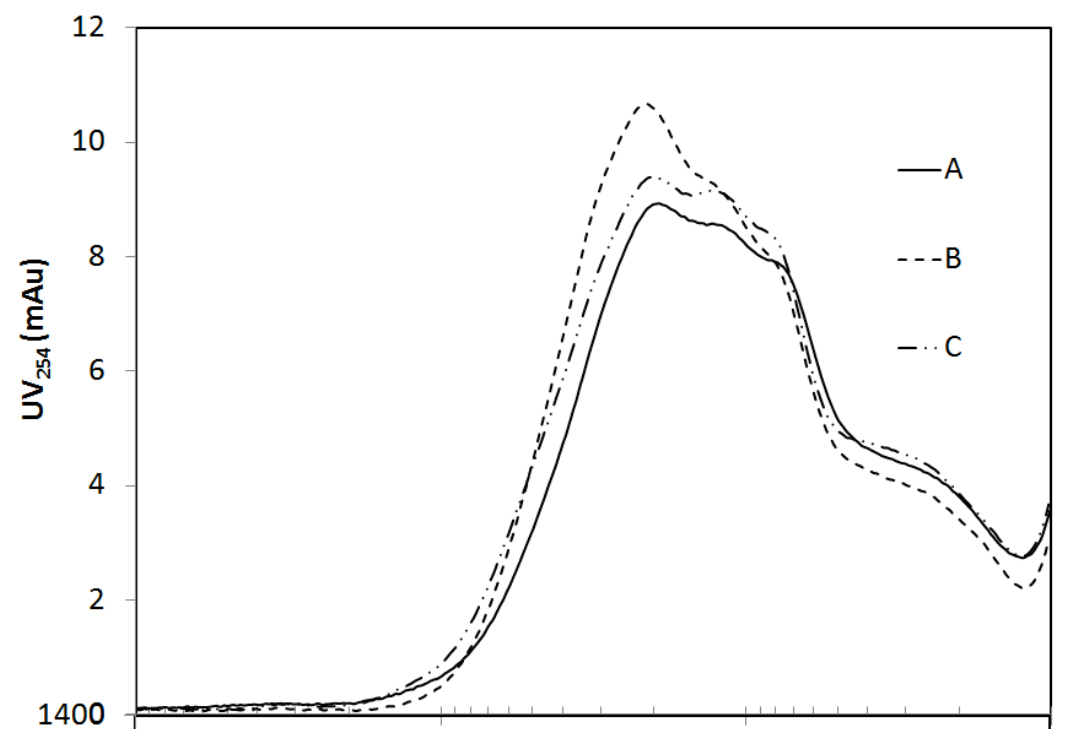

52

53

54

55

56

57

58

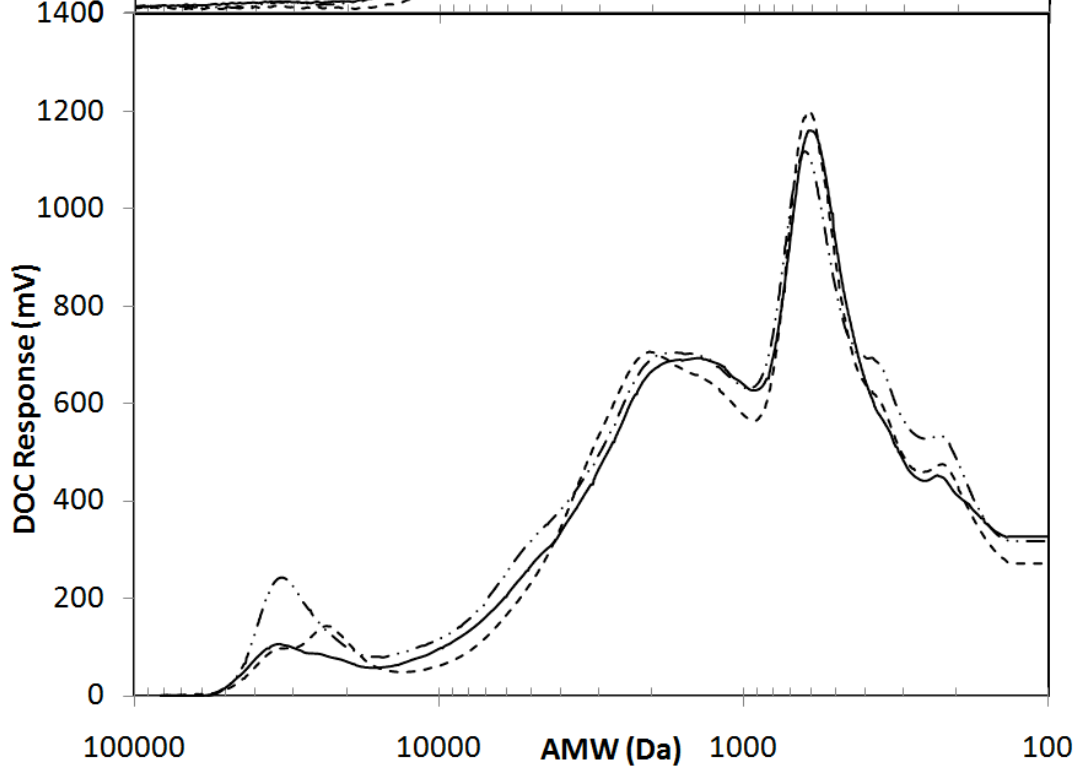

59

60

Figure S. 2. SEC of EfOM samples A-C. These SEC are typical for EfOM samples. ).

61 Conditions: buffer $\left(2.4 \mathrm{mM} \mathrm{NaH}_{2} \mathrm{PO}_{4}, 1.6 \mathrm{mM} \mathrm{Na}_{2} \mathrm{HPO}_{4}\right.$, and $25 \mathrm{mM} \mathrm{Na}_{2} \mathrm{SO}_{4}$ adjusted to pH 6.8

$62 \pm 0.1), 1.0 \mathrm{~mL} / \mathrm{min}$ flow rate, $2.0 \mu \mathrm{L} / \mathrm{min}$ acid and oxidizer flow rate. $\mathrm{UV}_{254}$ detection (top) and 63 DOC detection (bottom). 
65 Table S. 3. AMW estimations and $\boldsymbol{k}_{(\text {OM-Hо.) }}$ values.

\begin{tabular}{ccc}
\hline Sample & AMW (Da) & $k_{(\text {OM-HO. })} \times 10^{8}\left(\mathrm{M}_{\mathrm{C}}{ }^{-1} \mathrm{~s}^{-1}\right) 20{ }^{\circ} \mathrm{C}$ \\
\hline ESHA & 3100 & $1.21 \pm 0.09$ \\
SRFA & 2800 & $2.06 \pm 0.09$ \\
PLFA & 2400 & $6.90 \pm 0.53$ \\
Sample C & 2200 & $6.79 \pm 0.11$ \\
Sample A & 2100 & $7.39 \pm 0.06$ \\
& & \\
Sample B & 2100 & $9.37 \pm 0.07$ \\
\hline
\end{tabular}

66

67 
Table S. 4. Temperature Dependent rate data for all samples

\begin{tabular}{|c|c|c|}
\hline Sample & Temperature $\left({ }^{\circ} \mathrm{C}\right)$ & $k_{(\mathrm{OM}-\mathrm{HO} \cdot)} \times 10^{8}\left(\mathrm{M}_{\mathrm{C}}^{-1} \mathrm{~s}^{-1}\right)$ \\
\hline \multirow[t]{4}{*}{ ESHA } & 8.7 & $0.593 \pm 0.11$ \\
\hline & 24.4 & $1.21 \pm 0.09$ \\
\hline & 32.6 & $1.51 \pm 0.13$ \\
\hline & 42.0 & $2.36 \pm 0.12$ \\
\hline \multirow[t]{4}{*}{ SRFA } & 9.0 & $1.58 \pm 0.03$ \\
\hline & 20.6 & $2.06 \pm 0.09$ \\
\hline & 31.4 & $2.57 \pm 0.29$ \\
\hline & 43.3 & $3.06 \pm 0.10$ \\
\hline \multirow[t]{5}{*}{ PLFA } & 9.2 & $5.26 \pm 0.20$ \\
\hline & 14.7 & $5.92 \pm 0.36$ \\
\hline & 20.5 & $6.90 \pm 0.53$ \\
\hline & 29.9 & $8.30 \pm 0.69$ \\
\hline & 37.6 & $9.42 \pm 0.40$ \\
\hline \multirow[t]{2}{*}{ Sample A } & 9.7 & $5.56 \pm 0.23$ \\
\hline & 19.2 & $7.39 \pm 0.06$ \\
\hline
\end{tabular}




\begin{tabular}{|c|c|c|}
\hline & 30.9 & $9.02 \pm 0.33$ \\
\hline & 39.6 & $10.44 \pm 0.05$ \\
\hline \multirow[t]{5}{*}{ Sample B } & 9.4 & $7.25 \pm 0.28$ \\
\hline & 15.2 & $9.29 \pm 0.54$ \\
\hline & 19.4 & $9.37 \pm 0.07$ \\
\hline & 31.1 & $10.98 \pm 0.04$ \\
\hline & 39.6 & $11.80 \pm 0.04$ \\
\hline \multirow[t]{5}{*}{ Sample C } & 9.3 & $5.45 \pm 2.16$ \\
\hline & 15.0 & $6.27 \pm 0.21$ \\
\hline & 19.4 & $6.79 \pm 0.11$ \\
\hline & 29.7 & $9.18 \pm 0.80$ \\
\hline & 36.6 & $9.91 \pm 0.31$ \\
\hline
\end{tabular}

69

70

71

72

73 
74 References

75 1. Chin, M.; Whine, P., A temperature-dependent kinetics study of the aqueous phase 76 reactions $\mathrm{OH}+\mathrm{SCN}-\mathrm{SCNOH}-$ and SCN $+\mathrm{SCN}-(\mathrm{SCN}) 2-.$. J. Photochem. Photobiol. A-Chem. $771992,69,17-25$.

78 2. Buxton, G. V.; Wood, N. D.; Dyster, S., Ionisation constants of · OH and HO2 · in

79 aqueous solution up to $200^{\circ} \mathrm{C}$. A pulse radiolysis study. J. Chem. Soc., Faraday Trans. 1 1988, $80 \quad 84,1113-1121$.

$813 . \quad$ Hikel, B.; Sehested, K., Reaction of hydroxyl radicals with ammonia in liquid water at 82 elevated temperatures. . Radiat. Phys. Chem. 1992, 39, 355-357.

83

84 